\title{
Alzheimer Disease and Human Consciousness: A Neurogenetic Connection
} John K Grandy*

North Country Urgent Care, Watertown, N.Y, USA

*Corresponding author: John K. Grandy, North Country Urgent Care, Watertown, N.Y., USA, E-mail: khyber_john@yahoo.com

Received date: Feb 20, 2015; Accepted date: April 28, 2015; Published date: May 02, 2015

Copyright: @2015 Grandy JK. This is an open-access article distributed under the terms of the Creative Commons Attribution License, which permits unrestricted use, distribution, and reproduction in any medium, provided the original author and source are credited.

\begin{abstract}
Alzheimer disease (AD) is a neurodegenerative disorder that slowly erodes some of the facets of human consciousness. During the pathological course of AD, beta-amyloid plaques form which- causes damage to neurons and results in the gross loss of brain volume. As a consequence of $A D$, the afflicted individual develops decreases in cognitive/executive function, memory impairment/loss, and the inability to inhibit inappropriate behaviors. Another way of saying this is that the afflicted individual ceases to be the person whom they once were, i.e., their modalities of consciousness has deteriorated. Mutations in several genes, e.g., the gene variant APOE-epsilon4, APP, PSEN1, PSEN2, and TREM2 have all been correlated with the increased incidence and more rapid progression of AD. This has given support to the proposal that there are neurogenetic correlates of consciousness (NgCC). In previous works, these $\mathrm{NgCC}$ have been delineated into three neurogenetic phases of human consciousness. AD is a primary example of gene-based neurodegeneration that can take place in the third neurogenetic phase. There is hope that symptoms of $A D$ may be reversed with the development of novel genetic therapies. Some gene therapies are underway, e.g., FGF2, leptin, and NEU1 with the aim of reversing AD symptomatology. If these gene therapies are one day successful in reversing some of the symptoms of $A D$, can they eventually be used to enhance human consciousness in individuals without AD?
\end{abstract}

Keywords: Alzheimer disease; Human consciousness; Neurogenetic correlates of consciousness

\section{Introduction: Different Views of Human Consciousness}

In several academic fields, e.g., philosophy, psychology, and neuroscience- humankind has wrestled with the concept of consciousness. Defining consciousness can be difficult because it is studied by many different fields and there is no unilateral agreement across the board on how to define it. The difficulty defining consciousness has been discussed in other works that have been referenced for further inquiry [1-3]. I will, but very briefly, demonstrate only a few of the various views of consciousness.

Panpsychism is generally defined as a view in which all things possess mind or what one would consider a mind-like quality. This concept extends back to the ancient pre-Socratic Greek philosophers, e.g., Heraclitus $[4,5]$. Therefore it must be taken into consideration that the ancient Greeks did not have a word for consciousness per se. Hence, mind or mind-like quality would be synonymous with what most modern neurologists and consciousness researchers would refer to as consciousness. Moreover, pertaining to the definition of panpsychism, things typically refers to systems or collections of systems, e.g., cells or organisms. So in the panpsychist view all things in the universe possess a degree of consciousness.

There are some consciousness researchers who consider consciousness (called c-substrate) to be an element of the universe equal (and tethered) to space and time. This concept was originally defined by neuropsychiatrist Vernon Neppe and physicist Edward Close in their triadic dimensional vortical paradigm (TDVP) in where space-time-consciousness are tethered from the origin point of the universe and extend through mathematical dimensions $[6,7]$.
The views of panpyschism and the TVDP may differ from what a philosopher, a psychologist, or a neurobiologist may consider consciousness while objectifying a human model. For example, a philosopher whom is a supporter of dualism may view the mind and brain as two separate entities. Thus, according to a dualist, mind is not the same as brain because this philosophy maintains that there is a mental non-material world and a physical material world, i.e., there is dualism.

At this juncture, a basic and comprehensive definition of human consciousness could be- a collective totality of awareness, bodily (or somatic) sensations, perceptions (sensory input and neurobiological information), emotions, thoughts, and recollections of the self within a moment on the time continuum [1]. However, as previously mentioned, there are a sundry of other definitions of consciousness, which have been briefly discussed in the introduction, but now let us focus on a neurobiological definition of human consciousness.

A general neurobiological definition of human consciousness proposes three basic concepts: 1) consciousness emerges quite simply from an understanding of neuronal activity 2) consciousness is not a discrete operation of the brain, but rather, the outcome of computational activity of the associated areas of the brain 3) consciousness is a discrete phenomenon and that the issues of subjectivity, unity, and intentionality must be confronted if we are to understand how our experience is constructed [8].

Another analogous approach, known as the neural correlates of consciousness (NCC), was proposed by Francis Crick and Christopher Koch in 1990. By definition, NCC are the minimal neuronal mechanisms jointly sufficient for any one specific conscious principle or event [9]. It can be seen within this proposal that brain systems are active in tandem with the conscious experience. In addition, NCC can demonstrate that there are qualitative differences between the neural activity associated with the conscious and unconscious cognitive 
process [10]. Next I will briefly discuss four examples of NCC from the Toward a Science of Consciousness 2012 conference [11]:

- Areas of the brain that are affected by anesthesia, e.g., the frontal cortex integration to the posterior parietal cortex.

- Decreases in cerebral integration and connectivity to other areas of the brain. This has been demonstrated in studies using PET scans and functional MRI (fMRI) in patients with unresponsive wakefulness syndrome (also known as vegetative state) and in minimally conscious states.

- Frontoparietal connections in the brain that provide a global workspace. Two examples of these types of connections are: 1) lateral prefrontal and parietal cortices that function to provide external sensory awareness and 2) precuneal and mesiofrontal midline activity, which function to provide an internal awareness.

- Thalamo-cortical regions that have been shown to provide critically emergent properties of collective widespread connectivity of consciousness.

At this point we have, in a general sense, a neurobiological account of human consciousness that is based on NCC. However, the question needs to be asked- do we have the technology to validate this? As previously discussed there are numerous PET and fMRI studies that have focused on brain regions; specifically cerebral integration and connectivity between various areas of the brain, that are active during the conscious experience. However, more recently, a newer technique has been developed to determine levels of consciousness.

In a recent study, utilizing high-density electroencephalography (HD-EEG) signals that can be analyzed by a novel index called the perturbational complexity index (PCI) has been tested on the human brain [12]. This technique enables researchers to observe what happens to the brain electronically when it is responding to stimulus. This new EEG-derived index of human consciousness provides preliminary proof of electric correlates of consciousness. In addition, this new technique could become a simple noninvasive measurement to distinguish between conscious and unconscious states, for example: 1) in patients with brain damage- who display varying levels of consciousness 2) minimally conscious state 3) lock-in syndrome -a condition in where the patient is conscious but cannot move or talk.

What has been made clear with the discovery of NCC and corresponding studies with PET, fMRI, and now with HD-EEG is that human consciousness can be observed on a neurophysiologic pasture. Researchers now have a multimodal approach to observe what regions of the brain are stimulated during the conscious experience and how connectivity between brain regions is important. However, what is beneath this extravagant labyrinth of brain regions and interconnecting neurons? The answer is DNA. Let us now look at a neurogenetic account of human consciousness.

\section{A Neurogenetic Account of Human Consciousness}

Now that we have briefly reviewed a neurobiological account of human consciousness let us look at a deeper, even more perplexing, level known as the neurogenetic correlates of consciousness ( $\mathrm{NgCC}$ ). Up until now, only the emergent properties of neurons and function (and connectivity) of brain regions have been studied for their association with human consciousness. What the neurogenetic account of human consciousness proposes is that there is a need to look at the smaller scales of biology, in this case the NgCC.
The definition of NgCC is- genes or gene products (e.g., transcription factors or epigenetic factors) that have an effect on or are involved in the neurobiological process of human consciousness [13]. This approach is relatively new and it has been evaluated from both a neurological [14] and philosophical [15] point of view.

In this model, DNA gives rise to human consciousness, provides a continuum, and at the end of the lifespan can contribute to neuron degeneration, i.e., there are three neurogenetic phases of human consciousness. The focus on neurogenetics forces researchers to look beyond (or rather beneath) the brain and neurons as there is a rich neurogenetic substructure that supports human consciousness. Currently, this neurogenetic substructure is largely ignored by consciousness researchers or, by some, merely assumed to play a role in consciousness with the absence of a working paradigm. The neurogenetic account discussed in this work attempts to remedy these shortcomings in the neurobiological approach in consciousness research.

I will only very briefly describe the three neurogenetic phases of human consciousness. Far more detailed descriptions that delve into more neurogenetic elements can be found in previous publications $[13,16]$.

In the first neurogenetic phase there is an emergence of neuronbased consciousness. This begins at fertilization with master genes high in the developmental hierarchy that trans-activate genes downstream. This unfurls a highly sophisticated genetic cascade that depends on a nucleotide-based form of communication- that is based on two purines and two pyrimidines (sometimes referred to as DNA consciousness). There are several genes that give rise to brain regions involved in human consciousness- these are first phase NgCC. Here are some examples of $\mathrm{NgCC}$ in the first phase $[13,16]$ :

- Pax6- master gene for eye development and promotes the neurogenic fates of neural progenitor cells

- Otx1- influences $\sim 80 \%$ of the size of the cerebral cortex

- Otx2- is involved in diencephalon, mesencephalon, and telencephalon development

- Pax3- acts as a master gene early in development and influences several genes downstream, e.g., TP53, Hes1, Neurog2, and Meis2

In the second neurogenetic phase there is a continuum of neuronbased consciousness which requires the proper functioning of the genome. Like the first neurogenetic phase, it is also heavily reliant on the nucleotide-based communication system. The second neurogenetic phase can be studied objectively by observing the genetic basis of neuron plasticity (which is required for the continuum of human consciousness) and genetic abnormalities seen in certain psychiatric disorders, e.g., schizophrenia and autism. Here are some examples of genes involved in the second phase $[13,16]$ :

Neuron plasticity:

- BDNF, FGF2, delta-FosB, and synapsins I-III

\section{Genetic abnormalities in psychiatric disorders:}

- Autism- PTCHD1 locus disruptions

- Schizophrenia-associated genes- PDE4B, DISC1, and ZNF804a transcription factor

In the third neurogenetic phase there is neuron degeneration and loss of brain mass which leads to observable decreases in the degree of human consciousness. Keep in mind, neurodegeneration can be a 
normal age-related process as seen in mild cognitive impairment. However, it can have a genetic link as seen in diseases like Alzheimer disease (AD). Here are some genes associated with Alzheimer Disease in the third phase $[13,16]$ :

- APOE- $\varepsilon 4$ gene variant

- APP gene mutations

- PSEN1 gene mutations

- PSEN2 gene mutations

- TREM2 gene mutations

At this juncture a definition of the neurogenetic account of human consciousness and a survey of the three neurogenetic phases has been summarized. It must be kept in mind that the three neurogenetic phases of human consciousness is not intended to replace the NCC, nor any neurobiological account of human consciousness. Rather, the expectation is that the two systems are intimately connected and interface seamlessly, i.e., they are not two separate realms.

In the next section we will briefly discuss $\mathrm{AD}$ and the $\mathrm{NgCC}$ in the third phase. This will demonstrate a clear neurogenetic connection between human consciousness and a disorder like $\mathrm{AD}$; and in turn what $\mathrm{AD}$ can tell us neurologically and genetically about human consciousness. In addition, we will also take a look as some new genetic therapies aimed at treating $\mathrm{AD}$, which will further illustrate a neurogenetic connection to human consciousness.

\section{Alzheimer Disease and Human Consciousness}

$\mathrm{AD}$ is a neurodegenerative disease that is insidious and progressive, which ultimately results in irreversible cognitive impairment and is eventually fatal [17]. These cognitive impairments negatively affect the continuum of human consciousness. Here are some of the cardinal clinical features of $\mathrm{AD}[18]$ :

- Memory loss- remarkably in declarative memory

- Loss of cognitive skills- e.g. problem solving, language, calculation, and visuospatial perception

- Behavioral abnormalities- psychotic symptoms (e.g. hallucinations and delusions) and inappropriate behaviors (due to loss of inhibition)

Neuropsychological assessments that test attention span, memory, language skills, reasoning, planning, organization skills, and even the ability to self-reference on long-term episodic memory have demonstrated disease progression-related decline in $\mathrm{AD}$ patients $[19,20]$. Even a simple test that can make a gross assessment of consciousness, e.g., the Glasgow Coma Scale, has demonstrated that in $\mathrm{AD}$ patients there are decreases in the ability to respond to verbal command or painful stimuli, impairments in eliciting a verbal response, and impairments in motor responses [21].

During the advancement of $\mathrm{AD}$ there is progressive impairment in daily living and in the final stages patients can become mute, incontinent, and bedridden. At the later stage of $\mathrm{AD}$ the patient is completely reliant on a caregiver for even the most basic needs. In essence, the $\mathrm{AD}$ patient gradually losses cognitive viability as modalities of human consciousness are diminished. Eventually, they cease to be the person that they once were. Finally, one of the most devastating aspects of $\mathrm{AD}$ is that while the patient continues along the progressive path of diminution of mental activity, the loved ones and family members must lay witness to this heartbreaking transition.
The signs and symptoms of $\mathrm{AD}$ are directly related to the underlying pathophysiology which is primarily the accumulation in beta-amyloid plaques and the presence of neurofibrillary tangles that are composed of helical hyperphosphorylated tau protein. Regions of the brain associated with consciousness also demonstrate changes during the progression of AD. From a neurological standpoint, certain modalities of human consciousness are reliant on the function of various brain regions, e.g., the brain stem, basal forebrain, and diencephalic areas (thalamus and hypothalamus)- all of which support general arousal [22-26]. Neurophysiologic abnormalities have been demonstrated in these brain regions in patients with AD [27-29]. Furthermore, the functioning of the thalamocortical networks is crucial for the human brain to become aware of, and also to respond to, stimuli from both the external and internal environments [22,30-33]. These regions have demonstrated abnormalities in function and connectivity in patients with $\mathrm{AD}[34,35]$.

Therefore, we have a good amount of evidence that $\mathrm{AD}$ has a negative effect on several regions of the brain that are involved in modalities of human consciousness- primarily arousal and awareness. It was discussed earlier, that although the brain regions play a pivotal role in maintaining human consciousness, the underlying neurogenetic substructure should not be underestimated.

As discussed in the third neurogenetic phase of human consciousness, $\mathrm{AD}$ pathophysiology has genetic correlations, e.g., mutations in genes APOE, APP, PSEN1, PSEN2, and TREM2. The discovery of gene mutations in APP, PSEN1, and PSEN2 has enhanced our understanding of the underlying pathophysiology of $\mathrm{AD}$. However, more gene mutations are being identified, via, genome-wide studies and are currently being researched for their significance to $\mathrm{AD}$ [36]. I will briefly mention four:

- Bridging integrator 1 (BIN1) has been implicated as a late-onset AD susceptibility gene [37]. Currently it is unknown exactly what the role of BIN1 performs in $\mathrm{AD}$, but this study demonstrated that the largest BIN1 isoforms were reduced and the smaller isoforms were increased in the $\mathrm{AD}$ brain (hippocampus, inferior parietal cortex, inferior temporal cortex, frontal cortex area BA9, and the superior and middle temporal gyri) when compared to age-match controls.

- Clustering gene (CLU) mutations in major C allele rs11136000 and rs1532278 both demonstrated reduced CLU-expression, which has been associated with an increased risk for dementia and faster expansion of predictable longitudinal ventricular regions seen in $\mathrm{AD}$ [38]. Further analyses demonstrated that both CLU risk variants in combination with APOE- $\varepsilon 4$ gene variant had combined negative effects on both the volumetric expansion and lateral ventricle surface morphology.

- Phosphatidylinositol binding clathrin assembly protein (PICALM) gene single-nucleotide polymorphism rs3851179 has been implicated as an $\mathrm{AD}$ risk gene and has demonstrated synergistic adverse effects on both prefrontal brain atrophy and cognitive performance [39]. This was tested with Trail Making Test A which is sensitive to processing speed and working memory function.

- Complement receptor 1 (CR1) gene single-nucleotide polymorphism rs 3818361 is associated with a more rapid decline on the Annual Mini Mental State Examination testing scores in patients with sporadic AD [40]. Additionally, a meta-analysis demonstrated that CR1 single-nucleotide polymorphisms rs6656401 and rs3818361 have a significant association with lateonset AD susceptibility [41]. 
In terms of human consciousness, most of the signs and symptoms of $\mathrm{AD}$ are a perpetual assault on multiple modalities of human consciousness. For example, this can be seen most profoundly when one is no longer alert and orientated times three (AOX3), loses the ability to process and store new information, loses the ability to discriminate the environment (identify what objects are and their significance), demonstrates decreases in arousal/awareness, and the development of hallucinations and delusional thinking. Hallucinations and delusional thinking are important features because they represent the breakdown in the perception of reality- a disturbance in the continuum of human consciousness.

It is very profound that there is a neurogenetic connection between $\mathrm{AD}$ and human consciousness because now genetic abnormalities can be associated with disruptions and decreases in the continuum of human consciousness. Currently there are several gene candidates that play a discernable role in the neurogenetics of human consciousness. Since there is a genetic correlation to disorders like $\mathrm{AD}$, it would make prudent sense that genetic therapies may hold the keys to a cure.

\section{Gene Therapies for Treatment of AD}

Currently there are no treatments that are disease modifying agents or that can reverse the pathophysiology of AD. Most of the standard treatments merely decelerate the progression of the disease for a short period of time. These treatments provide, at best, marginal symptomatic benefits. Inevitably, a threshold is met when the accumulations of beta-amyloid and hyperphosphorylated tau protein inflict enough neuronal damage that it eventually results in gross brain loss. This is the end result of $\mathrm{AD}$ and it is irreversible. However, there may be some hope in genetic-based therapeutics. I will briefly mention three genetic therapies aimed at reversing some of the symptoms of $\mathrm{AD}$ :

- FGF2 transplant treatment to improve memory in AD [42]. In this study FGF2 gene was transferred directly to the hippocampus of bigenic mice (APP+presenilin-1). The FGF2 gene was delivered by an adeno-associated virus serotype $2 / 1$ hybrid. Significant improvement was seen in the mice receiving the injection at both the pre- and post-symptomatic stages of Alzheimer disease. This was verified by testing spatial learning in the radial arm water maze test.

- NEU1 Gene Therapy [43]. This study demonstrated that deficiencies of the lysosomal sialidase, which is produced by the NEU1 gene, leads to the spontaneous occurrence of an Alzheimerlike amyloidogenic process to occur in the brains of mice. In addition, this study subsequently demonstrated that cerebral injection of NEU1 in Alzheimer-mice resulted in substantial reduction of beta-amyloid plaques. This gives it potential to actually reverse one of the underlying pathologies of $\mathrm{AD}$.

- Leptin gene therapy [44]. This study demonstrated that leptin gene therapy was able to reduce the accumulation of beta-amyloid in the APP/PS1 transgenic mouse model. In this study, a lentivirus vector expressing leptin protein in a self-activating HIV-1 was delivered by intra-cerebroventricular administration. This resulted in a reduction of beta-amyloid accumulation and a partial rescue of synaptic density in these mice.

Earlier it was discussed that there is a significant connection between certain gene mutations and an association to $\mathrm{AD}$. In this section it can be seen that there is also a significant connection between gene therapy and the possibility of curing $\mathrm{AD}$ and rescuing function, e.g., memory, or potentially reversing the underlying pathophysiology. If gene therapies can restore modalities of human consciousness that were once taken away by AD pathophysiology, then this fortifies the neurogenetic connection to human consciousness. Another way of looking at this would be that, first there are certain gene mutations can decrease modalities of human consciousness as seen in $\mathrm{AD}$ and discussed in this paper. Secondly, there are several potential genetic therapies that may rescue or restore those modalities of consciousness. This is the neurogenetic connection!

At this juncture we must stop and ask ourselves, if genetic therapies; such as the ones briefly discussed in this paper, were to someday be successful, could they in turn be used on individuals without $\mathrm{AD}$ for sake of human enhancement? This is an important topic to broach as it summons many ethical questions; e.g., should neurogenetic enhancement be legal, how much enhancement should be allowed in humans, and who will have access to these types of therapies? In earlier publications the topic of genetic enhancements and the problems that could possibly ensue have been discussed [45]. In addition to the possibility of evolving human consciousness, neurogenetic therapies could lead to transhumanism, or perchance, posthumanism [46].

\section{Conclusion}

A brief survey of many different views of consciousness and the neurobiological approach to human consciousness was discussed. Some examples of NCC were summarized. It was pointed out that beneath this system of brain regions and neurons running in tandem with the conscious experience resides a hidden realm of NgCC. The three neurogenetic phases of consciousness were briefly outlined. It is in the third neurogenetic phase in where $\mathrm{AD}$ serves as an excellent example to illustrate the negative effects of neurodegeneration on the degrees of human consciousness. This is primarily because there are genetic correlates that have been identified, and more are being investigated, that can be associated with the breakdown of cognitive abilities and modalities of consciousness during the decline in the continuum of human consciousness. It has been demonstrated in this work that a neurodegenerative disease, e.g., $\mathrm{AD}$ can shed light on the understanding of human consciousness.

Even more significant is that it appears that the cure for $\mathrm{AD}$ seems to imply enhancement with genetic therapies, which if successful will fortify the proposal of a neurogenetic connection to human consciousness. Finally, it must also be taken under consideration that genetic therapies being developed to cure $\mathrm{AD}$ or to reverse the underlying pathophysiology, if one day successful, could also be used for human enhancement possibly opening the gates to transhumanism, and potentially, posthumanism. This possibility has largely been ignored and urgently needs to be discussed.

\section{References}

1. Grandy, John (2006) Consciousness. The Encyclopedia of Anthropology 1: 563-566.

2. Grandy, John (2009) Consciousness. In: The Encyclopedia of Time 1: 212-216.

3. Nunn, Chris (2009) Editor's Introduction: Defining Consciousness. The Journal of Consciousness Studies 16: 5-8.

4. Smith TV (1934) From Thales to Plato. Chicago: Chicago University Press.

5. Freeman, K. (1948) Ancilla to the Pre-Socratic Philosophers. Oxford: B. Blackwell. 
6. Neppe, Vernon, Edward R Close (2011) Applying Consciousness, Infinity, and Dimensionality Creating a Paradigm Shift: Introducing the Triadic Dimensional Distinction Vortical Paradigm. Neuro Quantology 3: 375-392.

7. Neppe, Vernon, Edward R. Close. (2013). Reality Begins with Consciousness: A Paradigm Shift That Works. Fourth Edition.

8. Kandel, Eric R, James H Schwartz, Thomas M Jessell, et al. (2013). Principles of Neural Science, 5th edition. McGraw Hill Page 385.

9. Francis Crick and Christof Koch (1990). Towards a neurobiological theory of consciousness. Seminars in the Neurosciences 2: 263-275.

10. Kandel, Eric R, James H Schwartz, Thomas M Jessell, et al. (2013). Principles of Neural Science, 5th edition. McGraw Hill Page 1375.

11. Grandy J (2012) The Explosion of Consciousness: TSC Conference Tucson Arizona 2012. Journal of Consciousness Exploration and Research 3: 432-445.

12. Casali AG, Gosseries O, Rosanova M, Boly M, Sarasso S, et al. (2013) A theoretically based index of consciousness independent of sensory processing and behavior. Sci Transl Med 5: 198ra105.

13. Grandy J (2013) The Neurogenetic Correlates of Consciousness, in R.L. Amoroso, L.H. Kauffman, \& P. Rowlands (eds.) 2013: The Physics of Reality: Space, Time, Matter, Cosmos, 8th Symposium in honor of JeanPierre Vigier Singapore: World Scientific. Chapter 48: 479-483.

14. Grandy J (2014) Neurogenetics and Human Consciousness. The Journal of Neurological Disorders 2: 1000e109.

15. Grandy J (2014) A Neurogenetic Account of Human Consciousness. Essays in Philosophy 15 (2).

16. Grandy J (2013). The Three Neurogenetic Phases of Human Consciousness. The Journal of Conscious Evolution 9: 1-24.

17. Alzheimer's Association (2014) 2014 Alzheimer's disease facts and figures. Alzheimers Dement 10: e47-92.

18. Kandel, Eric R, James H Schwartz, Thomas M Jessell, et al. (2013). Principles of Neural Science, 5th edition. McGraw Hill. Page 1335.

19. Weintraub S, Wicklund AH, Salmon DP (2012) The neuropsychological profile of Alzheimer disease. Cold Spring Harb Perspect Med 2: a006171.

20. Lalanne J, Rozenberg J, Grolleau P, Piolino P1 (2013) The self-reference effect on episodic memory recollection in young and older adults and Alzheimer's disease. Curr Alzheimer Res 10: 1107-1117.

21. Benesch CG, McDaniel KD, Cox C, Hamill RW (1993) End-stage Alzheimer's disease. Glasgow Coma Scale and the neurologic examination. Arch Neurol 50: 1309-1315.

22. Goldfine AM, Schiff ND (2011) Consciousness: its neurobiology and the major classes of impairment. Neurol Clin 29: 723-737.

23. Moruzzi G, Magoun HW (1949) Brain stem reticular formation and activation of the EEG. Electroencephalogr Clin Neurophysiol 1: 455-473.

24. Magoun, HW. (1958). The Waking Brain. Charles C. Thomas Publisher.

25. Parvizi J, Damasio A (2001) Consciousness and the brainstem. Cognition 79: 135-160.

26. Steriade M, Glenn LL (1982) Neocortical and caudate projections of intralaminar thalamic neurons and their synaptic excitation from midbrain reticular core. J Neurophysiol 48: 352-371.

27. Theofilas P, Dunlop S, Heinsen H, Grinberg LT (2015) Turning on the Light Within: Subcortical Nuclei of the Isodentritic Core and their Role in Alzheimer's Disease Pathogenesis. J Alzheimers Dis.
28. Cho H, Kim JH, Kim C, Ye BS, Kim HJ, et al. (2014) Shape changes of the basal ganglia and thalamus in Alzheimer's disease: a three-year longitudinal study. J Alzheimers Dis 40: 285-295.

29. Baker-Nigh A, Vahedi S, Davis EG, Weintraub S, Bigio EH, et al. (2015) Neuronal amyloid- $\hat{I}^{2}$ accumulation within cholinergic basal forebrain in ageing and Alzheimer's disease. Brain .

30. Llinás R, Ribary U, Contreras D, Pedroarena C (1998) The neuronal basis for consciousness. Philos Trans R Soc Lond B Biol Sci 353: 1841-1849.

31. Jones EG (2002) Thalamic circuitry and thalamocortical synchrony. Philos Trans R Soc Lond B Biol Sci 357: 1659-1673.

32. Tononi G, Edelman GM (1998) Consciousness and complexity. Science 282: 1846-1851.

33. Edelman Gerald M (2011) Biology of consciousness. Frontiers in Psychology 2: 1-7.

34. Abuhassan K, Coyle D, Maguire L (2014) Compensating for thalamocortical synaptic loss in Alzheimer's disease. Front Comput Neurosci 8: 65.

35. Zhou B, Liu Y, Zhang Z, An N, Yao H, et al. (2013) Impaired functional connectivity of the thalamus in Alzheimer's disease and mild cognitive impairment: a resting-state fMRI study. Curr Alzheimer Res 10: 754-766.

36. Chouraki V, Seshadri S (2014) Genetics of Alzheimer's disease. Adv Genet 87: 245-294.

37. Holler CJ, Davis PR, Beckett TL, Platt TL, Webb RL, et al. (2014). Bridging Integrator 1 (BIN1) Protein Expression Increases in the Alzheimer's Disease Brain and Correlates with Neurofibrillary Tangle Pathology. Journal of Alzheimer Disease 42: 1221-1227.

38. Schmidt C, Wolff M, von Ahsen N, Lange K, Friede T, et al. (2014) CR1 is potentially associated with rate of decline in sporadic Alzheimer's disease. J Clin Neurosci 21: 1705-1708.

39. Luo J, Li S, Qin X, Song L, Peng Q, et al. (2014) Meta-analysis of the association between CR1 polymorphisms and risk of late-onset Alzheimer's disease. Neurosci Lett 578: 165-170.

40. Kiyota T, Ingraham KL, Jacobsen MT, Xiong H, Ikezu T (2011) FGF2 gene transfer restores hippocampal functions in mouse models of Alzheimer's disease and has therapeutic implications for neurocognitive disorders. Proc Natl Acad Sci U S A 108: E1339-1348.

41. Annunziata I, Patterson A, Helton D, Hu H, Moshiach S, et al. (2013) Lysosomal NEU1 deficiency affects amyloid precursor protein levels and amyloid- $\hat{I}^{2}$ secretion via deregulated lysosomal exocytosis. Nat Commun 4: 2734.

42. Pérez-González R, Alvira-Botero MX2, Robayo O3, Antequera D1, Garzón M2, et al. (2014) Leptin gene therapy attenuates neuronal damages evoked by amyloid- $\hat{\mathrm{I}}^{2}$ and rescues memory deficits in APP/PS1 mice. Gene Ther 21: 298-308.

43. Grandy J (2010) Selected Genetic Destination and the Rise of Homo Sapiens Genomicus. The International Journal of Arts and Sciences 3: 166-190.

44. Grandy John (2014). The Three Neurogenetic Phases of Human Consciousness: The Possibility of Transhuman and Posthuman Consciousness. The International Journal of Arts and Sciences 7: 381-394. 